\title{
Original
}

\section{Papel actual de la linfadenectomia y la adrenalectomía en la cirugia radical del cáncer renal}

\author{
Emilio Ríos González, Luis Martínez-Piñeiro Lorenzo \\ Servicio de Urología. Hospital Infanta Sofia. San Sebastián de los Reyes, Madrid, España.
}

\begin{abstract}
Resumen
El único tratamiento con intención curativa del cáncer renal es la cirugía. La nefrectomía radical inicialmente incluía la adrenalectomía ipsilateral y la linfadenectomía regional. En los últimos años el desarrollo de la cirugía conservadora renal así como el diagnostico en un estadio cada vez más precoz de los tumores renales han llevado a plantearse la conveniencia de realizar tanto la linfadenectomía como la adrenalectomía. En esta revisión se recogen las indicaciones tanto de la linfadenectomía como de la suprarrenalectomía en el tratamiento quirúrgico del cáncer renal obtenidas tras una revisión de la literatura.
\end{abstract}

Palabras clave: Cáncer renal. Linfadenectomía. Adrenalectomía.

\section{Current role of lymphadenectomy and adrenalectomy in radical surgery for renal cancer Abstract}

Radical nephrectomy is the only curative treatment for renal cell cancer. Standard treatment includes ipsilateral adrenalectomy and lymph node dissection. In recent years, development of nephron-sparing surgery and early detection of small renal tumors has led to question this approach. The role of lymphadenectomy and adrenalectomy in surgical treatment of renal cancer is reviewed.

Keywords: Renal cell cancer. Lymphadenectomy. Adrenalectomy.

$\mathrm{E}$ 1 único tratamiento con intención curativa del cáncer renal es la cirugía. En 1969 Robson et al. ${ }^{1}$ describieron la técnica quirúrgica para llevar a cabo la nefrectomía radical. En dicha descripción se incluía la suprarrenalectomía ipsilateral y la linfadenectomía regional. La suprarrenalectomía se recomendaba por su sencillez relativa así como por la posibilidad de afectación de la misma por contigüidad y de afectación del margen quirúrgico por la proximidad del riñón. La linfadenectomía, según fue descrita incluía el tejido linfático paraaortico y paracavo desde la bifurcación de la aorta hasta pospilares diafragmáticos y se recomendaba por la creencia de que aumentaba la supervivencia. Con el paso de los años la utilidad de ambas ha sido puesta en duda. En esta revisión se recogen las indicaciones tanto de la linfadenectomía como de la suprarrenalectomía en el tratamiento quirúrgico del cáncer renal obtenidas tras una revisión de la literatura.

\section{LINFADENECTOMÍA}

El papel de la linfadenectomía en la cirugía del cáncer renal todavía genera controversia entre la mayoría de los urólogos. De hecho su realización como parte de la cirugía es criticada por varios autores a pesar de que el ensayo de la $\mathrm{EORTC}^{2}$, el único estudio prospectivo que compara nefrectomía radical con o sin linfadenectomía, demostró la ausencia de morbimortalidad de la misma.

Para definir la utilidad de la linfadenectomía en la cirugía del cáncer renal debemos tener en cuenta tres aspectos fundamentales:

1) La incidencia de metástasis ganglionares en los pacientes diagnosticados de cáncer renal y subsidiarios de tratamiento quirúrgico.

2) La morbilidad asociada a la misma.

3) El beneficio para el paciente.

\section{Incidencia}

La incidencia de metástasis linfáticas en el cáncer renal en series autópsicas y quirúrgicas es de aproximadamente el $20 \%{ }^{3}$. La mayoría de las series descritas en la literatura son retrospectivas y la incidencia oscila entre el $5 \%{ }^{4}$ y el $46 \%{ }^{5}$. Una exhaustiva revisión de las estudios retrospectivos fue llevada a cabo por Godoy et al. ${ }^{3}$ que objetivaron gran variabilidad en 
criterios de inclusión, diseño de los estudios, técnica quirúrgica empleada y extensión de la linfadenectomía. En series autópsicas la incidencia de afectación ganglionar varía entre el 14\% de la serie de Johnsen ${ }^{6}$ y el 63,6\% de la serie de Sahito ${ }^{7}$. El único estudio prospectivo es el llevado a cabo por la EORTC y la incidencia de afectación ganglionar descrita es del 3,3\%2.

Del análisis de las series publicadas se desprende que el estadio patológico y el grado de diferenciación tumoral son altamente predictivos de afectación ganglionar ${ }^{8-10}$, de manera que a mayor categoría $\mathrm{T}$ y mayor grado de Fuhrman, mayor es la incidencia de afectación ganglionar. Giuliani et $\mathrm{al}^{8}$ apreciaron afectación ganglionar en el 13,2\% de los pacientes con tumores pT1-2 y 36,1\% en tumores pT3-4. Hallazgos similares comunicaron Pantuck et al. ${ }^{9}$ que encontraron afectación ganglionar en el $5,2 \%$ de tumores pT1-2 frente al 23,4\% en tumores pT3-4. En este último estudio se encontró afectación linfática en el 33\% de los tumores con un grado de Fuhrman 1-2 y en el 68\% de los tumores con un grado de Fuhrman 3-4.

Con el paso de los años la incidencia de afectación ganglionar en las series quirúrgicas ha disminuido pasando de más del $30 \%$ de la serie de Robson $^{1}$ al $3,3 \%$ en la serie prospectiva de la EORTC $^{2}$. Un factor que puede explicar este hecho es el incremento en el diagnóstico de tumores incidentales que ha supuesto que estos se descubran en fases más precoces, con menor afectación ganglionar. En la serie de Skinner et al $^{11}$ entre 1935 y 1965 la incidencia de tumores incidentales era del 7\%. En series más recientes como la de Jayson y Sanders ${ }^{12}$, el porcentaje alcanzaba el 61\%. Konnak y Grossman también apreciaron un incremento en el diagnóstico de tumores incidentales del 13\% en los años 70 al $48 \%$ en los años 80

Con respecto a la importancia de la afectación ganglionar en el pronóstico y por tanto en la supervivencia caben destacar las series publicadas por Pantuck y Blute. En la serie de Blute ${ }^{13}$ la supervivencia cáncer específica a 5 años fue del 20,9\%. Esté último autor realizó un análisis multivariante en el que se aprecia que los pacientes con afectación ganglionar tienen 7,89 veces más probabilidades de morir de su enfermedad frente a los que no la tienen. En la serie de Pantuck ${ }^{9}$ la supervivencia cáncer específica a 5 años fue del 23\%. En esta serie los pacientes con afectación ganglionar a los que no se les realizó linfadenectomía presentaron 3 veces más probabilidad de morir por su enfermedad que aquellos a los que se les realizó.

\section{Morbilidad}

La técnica quirúrgica inicialmente descrita por Robson $^{1}$ incluía todo el tejido linfático paraaortico y paracavo desde el diafragma a la bifurcación iliaca. Posteriormente se describieron nuevos límites en función del lado del tumor. Así se recomendaba la linfadenectomía hiliar, paracava, precava, retrocava, interaórtica y preaórtica para los tumores derechos y la linfadenectomía hiliar, paraaórtica, preaórtica, retroaortica, interaortocava y precava para los tumores izquierdos ${ }^{14}$. En la práctica, muchos autores, en aras a disminuir la morbilidad de la disección, recomiendan una linfadenectomía regional limitada que incluye los ganglios paracavos, precavos e hiliares para el lado derecho y los paraaórticos, preaórticos e hiliares para el lado izquierdo ${ }^{3}$.

Las complicaciones más comúnmente asociadas a la linfadenectomía en el cáncer renal han sido el linfocele, la ascitis quilosa, el sangrado de vasos lumbares o grandes vasos y las lesiones de órganos adyacentes ${ }^{3}$; sin embargo, los estudios realizados que comparaban nefrectomía y linfadenectomía frente a nefrectomía sola no han demostrado diferencias significativas en cuanto a complicaciones entre los dos grupos ${ }^{2,9,15}$. El estudio de la EORTC sólo demostró un mayor sangrado quirúrgico en los pacientes a los que se les realizaba linfadenectomía ${ }^{2}$. A pesar de que en la literatura no se han demostrado diferencias en morbilidad entre realizar linfadenectomía o no, la mayoría de los autores la consideran un técnica quirúrgica con un gran riesgo potencial de complicaciones intraoperaotrias ${ }^{3}$.

\section{Beneficios}

A lo largo de los años se han evaluado tres potenciales beneficios de la linfadenectomía en la cirugía del cáncer renal:

1) Mejorar la estadificación y el pronóstico,

2) Mejorar la supervivencia y

3) Mejorar la respuesta a tratamientos sistémicos. A la hora de evaluar los beneficios de la linfadenectomía en el cáncer renal es preciso distinguir entre:

- Pacientes con tumores localizados.

- Pacientes con afectación ganglionar.

- Pacientes con afectación ganglionar y metástasis a distancia. 
Aunque las modernas técnicas de imagen son capaces de detectar ganglios linfáticos de hasta 5 $\mathrm{mm}$, éstas no son capaces de diferenciar entre ganglios inflamatorios y metastásicos en el cáncer renal $^{16,17}$. Studer ${ }^{18}$ publicó una serie en la que sólo el $42 \%$ de los pacientes con sospecha preoperatoria de metástasis ganglionar en la TAC presentaban confirmación histológica. Este autor concluyó que los ganglios detectados mediante TAC con un tamaño entre 1 y $2,2 \mathrm{~cm}$ tenían más posibilidades de ser inflamatorios que tumorales.

Pacientes con tumores localizados: La realización de linfadenectomía en pacientes con tumores clínicamente localizados no ha demostrado beneficio significativo. La baja incidencia de afectación ganglionar en este tipo de tumores $\left(0,4 \%-3,3 \%^{2,4,9}\right)$ y la ausencia de beneficio demostrado en el estudio de la EORTC $^{2}$ desaconsejan su realización sistemática. Blute y colaboradores ${ }^{13}$ desarrollaron un modelo predictivo con el fin de identificar a pacientes diagnosticados de cáncer renal no metastático que tendrían más probabilidad de presentar afectación ganglionar. En el estudio multivariante identificaron 5 factores de riesgo que se comportaban como factores predictivos independientes de afectación ganglionar:

1) Categoría $T$ (T3-T4).

2) Tamaño del tumor $(>10 \mathrm{~cm})$.

3) Grado de Fuhrman (3-4).

4) Presencia de necrosis.

5) Presencia de diferenciación sarcomatoide.

La presencia de dos o más de estos factores se asociaba con una probabilidad 15 veces mayor de padecer metástasis ganglionar. La limitación de este estudio es que la mayoría de los factores de riesgo no están disponibles en el momento de la cirugía.

\section{Pacientes con afectación ganglionar}

Estos pacientes representan aproximadamente el 0,9-10\% de los casos según las series ${ }^{2,4,6,8,9}$ Terrone et $\mathrm{al}^{17}$ demostraron que cuando realizaban linfadenectomía extendida y recuperaban más de 12 ganglios linfáticos la incidencia de afectación ganglionar era mayor $(20,4 \%)$. La supervivencia de los pacientes tratados con nefrectomía y linfadenectomía fue superior a los que fueron tratados con nefrectomía sola en este grupo de pacientes. La supervivencia de los pacientes sometidos a linfadenectomía fue muy superior a la de los que presentan metástasis y cercana a la de los pacientes con tumores pT3NOMO. Giuliani et al. ${ }^{8}$ encontraron tasas de supervivencia para este grupo de pacientes del $47,9 \%$ y $31,9 \%$ a 5 y 10 años respectivamente en comparación con el 7\% de supervivencia a 5 años de los pacientes con metástasis a distancia. Peters y Brown ${ }^{19}$ demostraron un incremento en las tasas de supervivencia en los pacientes a los que realizaban linfadenectomía del $56,5 \%$ al $87,5 \%$ a un año y del $25,79 \%$ al $43,75 \%$ a 5 años. La aceptable morbilidad de la linfadenectomía nos puede llevar a realizarla en todos los casos en los que encontremos ganglios. Hemos de tener en cuenta que el estudio de la EORTC $^{2}$ demostró que sólo el 16\% de los ganglios sospechosos son realmente metastáticos y sólo lo fueron en el 1\% en aquellos casos en que la linfadenectomía se realizó de forma rutinaria.

Pacientes con afectación ganglionar y metástasis a distancia

La reciente aparición de inhibidores multiquinasa en el tratamiento del cáncer renal diseminado ha supuesto una revolución en el tratamiento de esta enfermedad. Estudios previos han demostrado que el tratamiento sistémico con citoquinas mejora la supervivencia tras nefrectomía radical y la respuesta de estos pacientes a la quimioterapia e inmunoterapia es peor en presencia de adenopatías ${ }^{3,5,9}$. Vaselli et al. ${ }^{5}$ observaron que la supervivencia en pacientes con enfermedad diseminada sin afectación ganglionar era mejor que en aquellos que sí la presentaban. Además, cuando las adenopatías eran resecadas durante el acto quirúrgico la supervivencia de estos pacientes era similar a la del grupo con enfermedad diseminada sin adenopatías.

\section{Recomendaciones}

Godoy et al. ${ }^{3}$ en una reciente publicación han sintetizado un algoritmo que resume las indicaciones reales de linfadenectomía en la cirugía del cáncer renal hoy en día:

Pacientes con enfermedad localizada y de bajo riesgo (T1-2NOMO): No existe indicación de linfadenectomía.

Pacientes en enfermedad localizada y de alto riesgo (T3-4NOMO): Recomiendan la realización de linfadenectomía en aquellos pacientes en los que se objetiven adenopatías macroscópicas durante el acto quirúrgico adecuando su extensión a la comorbilidad y el estado general del paciente. 
Pacientes con metástasis ganglionares (T1-4N+ MO): Se recomienda la realización de linfadenectomía extendida.

Pacientes con enfermedad metastásica (T1-4N+ $M+)$ : Se recomienda cirugía con linfadenectomía regional en aquellos pacientes candidatos a citorreducción y tratamiento sistémico posterior.

\section{Conclusión}

Hoy en día sólo hay dos situaciones en las que el paciente se beneficie de la realización de linfadenectomía asociada a nefrectomía en el cáncer renal:

1) Afectación ganglionar preoperatoria sin metástasis a distancia

2) Enfermedad diseminada como parte de la cirugía citorreductora previa a tratamiento sistémico.

\section{ADRENALECTOMÍA}

La adrenalectomía ipsilateral en el transcurso de la nefrectomía en la cirugía del cáncer renal es un procedimiento sencillo y que en un porcentaje de casos se hace necesario debido a la lesión iatrogénica de la glándula durante el acto quirúrgico. Por otro lado el bajo riesgo de metástasis adrenal ipsilateral junto al diagnostico en un estadio cada vez más precoz hacen que día a día el tratamiento conservador sea una realidad ${ }^{20}$.

Al igual que en el caso de la linfadenectomía, para definir la utilidad de la suprarrenalectomía en la cirugía del cáncer renal debemos tener en cuenta tres aspectos fundamentales:

1) La incidencia de afectación suprarrenal en los pacientes diagnosticados de cáncer renal y subsidiarios de tratamiento quirúrgico,

2) La morbilidad asociada a la misma y

3) El beneficio para el paciente.

\section{Incidencia}

La afectación de la glándula ipsilateral habitualmente se relaciona con la proximidad al riñón asî como por la existencia de un drenaje venoso y linfático común, principalmente en el lado izquierdo ${ }^{21}$. La incidencia de afectación suprarrenal en el cáncer renal oscila entre el 1,2 y el 10\% en las series quirúrgicas ${ }^{20,22-27}$. Este porcentaje se incrementa en las series autopsias de pacientes con enfermedad diseminada, situándose entre el 7 y el $23 \% \%^{22,28}$. Sin embargo, la afectación de la glándula contralateral es muy rara ${ }^{29}$. También es relativamente infrecuen- te el hallazgo de metástasis adrenal solitaria en autopsias de pacientes con enfermedad diseminada $(2,8 \%)^{23}$.

La categoría $\mathrm{T}$, el tamaño, el lado izquierdo y la afectación del polo superior se han implicado en la aparición del afectación suprarrenal.

Von Knobloch ${ }^{30}$ en un estudio sobre 589 pacientes encontró afectación suprarrenal en el 0,9\% de los tumores hasta T2 y del 6,6\% en los tumores T3T4. Hallazgos similares encontraron Shalev ${ }^{23} \mathrm{y}$ Siemer ${ }^{31}$; en sus series todos los pacientes con afectación suprarrenal presentaban tumores T3N1 o superior. En la serie publicada por de $\mathrm{Sio}^{22}$ los pacientes con tumores $\mathrm{T} 1$ presentaron afectación suprarrenal en el 1,3\% frente al 6,4\% en los tumores T3-4. En esta misma serie no se encontró afectación suprarrenal en tumores sup>32 en su serie de 819 pacientes encontró metástasis suprarrenal en 6 pacientes con tumores entre 5 y $7 \mathrm{~cm}$ en 3 con tumores entre 2,5 y $5 \mathrm{~cm}$ y ninguna en pacientes con tumores $<2,5 \mathrm{~cm}$.

Con respecto a que la localización del tumor en el polo superior favorecería la afectación suprarrenal por contigüidad, los hallazgos en la literatura son contradictorios. Mientras que Tsui ${ }^{25}$ encontró que la afectación del polo superior era predictora de afectación suprarrenal, von Knobloch ${ }^{30}$ y Wunderlich $^{33}$ no encontraron esta asociación. Similares resultados encontramos al estudiar la influencia de la afectación del lado izquierdo como predictor de afectación suprarrenal. La serie de Leibovith $^{34}$ demuestra una alta incidencia de afectación suprarrenal en tumores izquierdos mientras que la de Shalev $^{23}$ y von Knobloch ${ }^{30}$ demuestran afectación suprarrenal derecha en el 73 y 58\% de los casos respectivamente. Tsui ${ }^{25}$ en su serie comunica una alta incidencia de metástasis suprarrenal en el lado izquierdo sólo en presencia de trombosis de la vena renal.

Kuczyk $^{32}$ en su serie demostró, en el estudio multivariante, que la presencia de metástasis a distancia, el crecimiento multifocal y la invasión vascular en el tumor primario se comportaban como factores pronósticos independientes de afectación suprarrenal. Paul ${ }^{20}$ en su serie demostró que la presencia de metástasis a distancia y el tamaño tumoral mayor de $8 \mathrm{~cm}$ se comportaban igualmente como factores pronósticos independientes de afectación suprarrenal. En un análisis retrospectivo recientemente publicado por la Sociedad Japonesa de Cáncer Renal ${ }^{35}$ han 
demostrado que el tamaño tumoral mayor de $5,5 \mathrm{~cm}$ el estadio clínico T3 o superior y la presencia de metástasis ganglionar o a distancia se comportan como factores predictivos independientes de afectación adrenal ipsilateral.

\section{Morbilidad}

El estudio publicado por Hellstrom ${ }^{36}$ evidenció que la realización de adrenalectomía y nefrectomía no añadía comorbilidad significativa ni prolongaba el tiempo quirúrgico frente a la nefrectomía sola.

Al ser la adrenal una glándula par, cabe pensar que la extirpación de una de ellas no conllevaría pérdida de función. No obstante algunos autores ${ }^{22}$ han evidenciado que hasta un $20 \%$ de los pacientes sometidos a nefrectomía y adrenalectomía desarrollan insuficiencia adrenal. Yokohama y Tanaka ${ }^{26}$ demostraron que los pacientes sometidos a adrenalectomía presentaban a las dos semanas un incremento en los niveles de ACTH y una disminución significativa de los niveles de cortisol en plasma frente a aquellos a los que no se le realizaba la limitación de este estudio es que no aporta resultados de función adrenal a largo plazo.

\section{Beneficios}

Robey y Schellhammer ${ }^{37}$ estudiaron a 52 pacientes con cáncer renal del los cuales 25 habían sido sometidos a adrenalectomía. No encontraron diferencias significativas en las tasas de supervivencia a 5 y 9 años entre ambos grupos. Leibovitch ${ }^{34}$ encontró similares hallazgos en su serie de 109 pacientes, 55 sin adrenalectomía. Kozak objetivó metástasis en 8 pacientes de su serie de 109 pacientes, los cuales fallecieron con una media de seguimiento de 15 meses sin objetivar efecto curativo de la adrenalectomía. En la serie de Sagalowsky ${ }^{38}$ de 695 pacientes con cáncer renal, de los 30 pacientes con afectación suprarrenal sólo 3 estaban vivos después de 27 meses. Parece por tanto que la preservación de la glándula suprarrenal ipsilateral no influye en la recurrencia del tumor ni en las tasas de supervivencia en comparación con la nefrectomía radical estándar. Sólo se beneficiarían de la adrenalectomía aquellos pacientes con afectación suprarrenal aislada sin enfermedad diseminada. Lamentablemente la incidencia de afectación suprarrenal aislada es rara. Wundwerlich ${ }^{33}$ encontró que de los pacientes con afectación suprarrenal la mayoría presenta enfermedad diseminada al diag- nóstico al tratarse de tumores de alto grado. En la serie de Paul $^{20}$ sobre 866 pacientes, sólo el 0,7\% de los mismos presentan metástasis suprarrenal aislada. Resultados similares describe Martínez Piñeiro ${ }^{27}$ en su serie donde la afectación suprarrenal aislada constituye el 0,7\% de los casos.

El diagnóstico preoperatorio de afectación adrenal, metástatica o por contigüidad, aislada o en el contexto de enfermedad diseminada es de capital importancia a la hora de determinar la realización o no de adrenalectomía ipsilateral. La TAC es hoy en día la prueba de imagen de elección para el diagnostico de lesiones adrenales y su caracterización ${ }^{22}$. Aunque el tamaño de la lesión puede orientar sobre su comportamiento benigno o maligno, éste no debe ser el único dato a tener en cuanta. De hecho, se considera que lesiones adrenales menores de $3 \mathrm{~cm}$ suelen tener un comportamiento benigno (adenoma, feocrocitoma) ${ }^{22,25}$ pero en la serie de Tsui ${ }^{25}$ el tamano medio de las lesiones adrenales secundarias a adenocarcinoma renal fue de $3,86 \mathrm{~cm}$. Por tanto este autor sugiere que aunque la grandes masas adrenales, sobre todo por encima de $6 \mathrm{~cm}$ suelen ser metástasis del tumor renal primario, no debe ser el tamaño el único factor a tener en cuenta de cara a la caracterización de las mismas. En la TAC realizada para valorar las lesiones adrenales existen dos factores a tener en cuenta: su contenido en grasa y sus propiedades vasculares. Las lesiones adrenales benignas (adenomas, mielolipomas,...) suelen tener un alto contenido en grasa y suelen ser de baja densidad $^{39}$. Lee ${ }^{40}$ fue de los primeros en documentar la utilidad de la TAC sin contraste y el coeficiente de atenuación en la caracterización de las lesiones suprarrenales. Actualmente se acepta un valor de corte de 10 unidades Hounsfield en el estudio sin contraste por debajo del cual las lesiones serían consideradas benignas ${ }^{39}$. En los estudios con contraste no es posible discernir lesiones benignas de malignas según su coeficiente de atenuación aunque se ha observado de forma significativa una más rápida eliminación del contraste en adenomas que en lesiones metastáticas.

\section{Recomendaciones}

En la Tabla 1 se recogen las indicaciones de adrenalectomía sugeridas por diversos autores. En la mayoría de los casos el tamaño tumoral y los hallazgos preoperatorios de la TAC determinan la realización o no de adrenalectomía asociada a nefrectomía. 
Tabla 1. Recomendaciones de adrenalectomía.

\begin{tabular}{|c|c|c|c|}
\hline Autor & $N^{\circ}$ de pacientes & Metástasis adrenal & Recomendaciones adrenalectomia \\
\hline Winter $(1990)^{21}$ & 138 & $5,8 \%$ & En todos los casos \\
\hline Martínez-Piñeiro (1990) ${ }^{27}$ & 198 & $2,7 \%$ & Tumores grandes o de polo superior. TAC anormal. \\
\hline Sagalowsky $(1994)^{38}$ & 695 & $4,3 \%$ & Adrenal macroscópicamente anormal \\
\hline Gill $(1994)^{41}$ & 157 & $6,4 \%$ & TAC anormal \\
\hline Shalev $(1995)^{23}$ & 285 & $3,8 \%$ & Sospecha intraoperatoria \\
\hline Kozak $(1996)^{42}$ & 225 & $3,5 \%$ & TAC anormal. Margen con el parénquima de $1 \mathrm{~cm}$ \\
\hline Wunderlich (1999) ${ }^{33}$ & 639 & $1,4 \%$ & TAC anormal. Adrenal macroscópicamente anormal \\
\hline Von Knobloch (1999) ${ }^{30}$ & 589 & $3,2 \%$ & Todos los casos \\
\hline Tsui $(2000)^{25}$ & 511 & $5,7 \%$ & $\begin{array}{l}\text { Factores de riesgo: Alto grado, multifocal, polo superior, } \\
\text { trombosis vena renal }\end{array}$ \\
\hline Paul $(2001)^{20}$ & 866 & $3,2 \%$ & TAC anormal. Tumores $>$ de $6 \mathrm{~cm}$ \\
\hline Kuczyk $(2002)^{32}$ & 819 & $3,3 \%$ & TAC anormal. Adrenal macroscópicamente anormal \\
\hline De Sio $(2003)^{22}$ & 201 & $4,4 \%$ & Tumores grandes o de alto riesgo (no en $\mathrm{T} 1-2$ ) \\
\hline Kobayashi $(2003)^{24}$ & 393 & $3 \%$ & Tumores localmente avanzados. TAC anormal. \\
\hline Yokohama $(2004)^{26}$ & 247 & $2,8 \%$ & $\begin{array}{l}\text { No en pacientes con estadio clínico bajo ni con TAC } \\
\text { normal }\end{array}$ \\
\hline Ito $(2008)^{35}$ & 956 & $3,1 \%$ & Tumores $>5,5 \mathrm{~cm}$. Estadio T3 o superior. TAC anormal \\
\hline
\end{tabular}

\section{Conclusiones}

Hoy en día se recomienda realizar adrenalectomía asociada a nefrectomía en las siguientes situaciones:

1) Tumores de tamaño superior a 5,5-6 cm.

2) Tumores en los que la TAC preoperatoria sugiera afectación suprarrenal.

3) Tumores con metástasis adrenal solitaria.

En el resto de las situaciones está indicada la preservación de la suprarrenal ipsilateral.

\section{REFERENCIAS}

1. Robson CJ, Churchill BM, Anderson W. The results of radical nephrectomy for renal cell carcinoma. J Urol. 1969;101(3):297-301.

2. Blom JHM, vanPoppel H, Marechal JM, Jacqmin D, Sylvester R, Schröeder FH, et al. Radical nephrectomy with and whitout lymph node disecction: preliminary results of the EORTC randomized phase III protocol 30881. Eur Urol. 1999;36(6):570-575.

3. Godoy G, O’Malley RL, Taneja SS. Lymph node dissection during de surgical treatment of renal cancer $y$ the modern area. International Braz J Urol. 2008;34(2):132-142.

4. Minervini A, Lilas L, Morelli C, Traversi S, Battaglia R, Cristofani $\mathrm{R}$, Minervini R. Regional lymph node disection in the treatment of renal cell carcinoma: is it useful in patiens with no suspected adenopathy before or during surgery?. BJU International 2001; 88: 172.

5. Vaselli JR, Yang JC, Linehan WM, White DE, Rosenberg SA, Walther MM. Lack of retroperitoneal lymphadenopathy predicts survival of patiens with metastatic cell carcinoma. J Urol. 2001; 166: 72 .
6. Johnsen JA, Hellsten S. Lymphatogenous spread of renal cell carcinoma: an autopsy study. J Urol.1997;157(2):450-453.

7. Saitoh H, Nakayama M, Nakamura K, Satoh T. Distant metastasis of renal adenocarcinoma in nephrectomized cases. J Urol.1982;127(6):1092-1095.

8. Giuliani L, Giberti C, Martorana G, Rovida S. Radical extensive surgery for renal cell carcinoma: long-term results and pronostic factors. J Urol. 1990;143(3):468-73.

9. Pantuck A, Zisman A, Dorey F, Chao DH, Han K, Said J, et al. Renal cell carcinoma with retroperitoneal lymph nodes: Role of lymph node dissection. J Urol. 2003;169(6):2076-2083.

10. Phillips CK, Taneja SS. The role of lymphadenectomy in the surgical managementof renal cell carcinoma. Urol Oncol. 2004;22(3):214-23.

11. Skinner DG, Colvin RB, Vermillion CD, Pfister RC, Leadbetter WF. Diagnosis a management of renal cell carcinoma. A clinical and pathological study of 309 cases. Cancer. 1971;28(5): 1165-77.

12. Jayson M, Sanders H. Increased incidence or serendipitously discovered renal cell carcinoma. Urology. 1998;51(2):203-205.

13. Blute ML, Leibovich BC, Cheville JC, Lohse CM, Zincke H. A protocol for performing extended lymph node disssection using primary tumor pathological features for patiens treated with radical nepherctomy for clear cell renal cell carcinoma. J Urol. 2004;172(2):465-469.

14. Wood DP Jr. Role of lymphademectomy in renal cell carcinoma. Urol Clin North Am. 1991;18(3):421-426.

15. Freedland SJ, deKernion JB. Role of lymphadenectomy for patiens undergoing radical nephrectomy for renal cell carcinoma. Rev Urol. 2003;5(3):191-195.

16. Bechtold RE, Zagoria RJ. Imaging approach to staging of renal cell carcinoma. Urol Clin North Am. 1997;24(3):507-522.

17. Terrone C, Guercio S, De Luca S, Poggio M, castelli E, Scoffone $\mathrm{C}$, et al. The number of lymph nodes examined and staging accuracy in renal cell carcinoma. BJU Int. 2003;91(1):37-40. 
18. Studer UE, Scherz S, Scheidegger J, Kraft R, Sonntag R, Ackermann D, et al. Enlargement of regional lymph nodes in renal cell carcinoma is often not due to metastasis. J Urol. 1990;144(2 Pt 1):243-245.

19. Peters PC, Brown GL. The role of lymphadenectomy in the management of renal cell carcinoma. Urol Clin North Am. 1980;7(3):705-709.

20. Paul R, Mordhost J, Busch R, Leyh H, Rartung R. Adrenal sparing surgery during radical nephrectomy in patiens with renal cell cancer: a new algorithm. J Urol. 2001;166(1):59-62.

21. Winter P, Miersch WD, Vogel J, Jaeger N. On the necessity of adrenal extirpation combined with radical nephrectomy. J Urol. 1990;144(4):842-843.

22. de Sio M, Autorino R, Di Lorenzo G, Damiano R, Cosentino L, De Placido S, et al. Adrenalectomy: Defining its role in the surgical treatment of renal cell carcinoma. Urol Int. 2003;71(4): 361-367.

23. Shalev M, Cipolla B, Guille F, Staerman F, Lobel B. Is ipsilateral adrenalectomy a neccesary componet of radical nephrectomy?. J Urol. 1995;153(5):1415-1417.

24. Kobayashi T, Nakamura E, YAmamoto S, Kamoto T, Okuno H, Terai A, et al. Low incidence of ipsilateral adrenal involvement and recurrences in patiens with renal cell caecinoma undergoing radical nephrectomy: A retrospective analysis of 393 patiens. Urology. 2003;62(1):40-45.

25. Tsui K, Shvarts O, Barbaric Z, Figlin R, deKernion JB, Belldegrun A. Is adrenalectomy a necessary component of radical nephrectomy? UCLA experiencie with 511 radical nephrectomies. J Urol. 2000;163(2):437-441.

26. Yokohama H, Tanaka M. Incidence of adrenal involvement and assessing adrenal function in patiens with renal cell carcinoma: Is ipsilateral adrenalectomy indispensable during radical nephrectomy?. BJU Int. 2004;95:526-529.

27. Martinez Piñeiro L, Lopez Ferrer P, Ortin M, Hidalgo L, Gaston de Iriarte E, Martinez Piñeiro JA. ¿Es necesario asociar en el tratmaiento quirúrgico del adenocarcinoma renal la adrenalectomía ipsilateral?. Arch Esp Urol. 1990;43(2):147-152.

27. Martinez Piñeiro L, Lopez Ferrer P, Ortin M, Hidalgo L, Gaston de Iriarte E, Martinez Piñeiro JA. Is it necessary to combine the surgical treatment of kidney adenocarcinoma with ipsilateral adrenalectomy?. Arch Esp Urol. 1990;43(2):147-152.

28. Saitoh H, Hida M, Nakamura K, Shimbo T, Shiramizu T, Satoh T. Metastatic processes and a potential indication of treatment for metastatic lesions of renal adenocarcinoma. J Urol. 1982; 128(5):916-918.

29. Lemmers M, Ward K, Hatch T, Stenzel P. Renal adenocarcinoma with solitary to the contralateral adrenal gland: report of two cases and review of the literature. J Urol. 1989;141(5): 1177 .

30. von Knobloch R, Seseke R, Riedmiller H, Grone HJ, Walthers EM, Kalble T. Radical nephrectomy for renal cell carcinoma: Is adrenalectomy necessary?. Eur Urol. 1999;36(4):308.
31. Siemer S, Lehmann J, Kamradt J, Remberger K, Hunke U, Ziegler M, et al. Adrenal metastases in 1635 patiens with renal cell carcinoma. Outcome and indication for adrenalectomy. J Urol. 2004;171(6 Pt 1):2155-2159.

32. Kuczyk M, Münch T, Machtens S, Bokemeyer C, Wefer A, Hartmann J, et al. The need for routine adrenalectomy during surgical treatment for renal cell cancer: The Hannover experience. BJU Int. 2002;89(6):517-522.

33. Wunderlich H, Schlichter A, Reichelt O, Zermann DH, Janitzky V, Schubert J. Real indications for adrenalectomy in renal cell carcinoma. Eur Urol. 1999;35(4):272-276.

34. Leibobitch I, Raviv G, Mor Y, Nativ O, Goldwasser B. Reconsidering the necessity of ipsilateral adrenalectomy during radical nephrectomy for renall cell carcinoma. Urology. 1995;46(3):316-320.

35. Ito K, Nakazawa H, Marumo K, Ozono S, Igarashi T, Shinohara $\mathrm{N}$, et al. Risk factors for ipsilateral adrenal involvement in renal cell carcinoma. Urology. 2008;72(2):354-358.

36. Hellstrom PA, Blogiu R, Roukonen AO, Vainionpaa VA, Nuutinen LS, Kontturi MJ. Is routine ipsilateral adrenalectomy during radial nephrectomy harmful of the patien?. Scand J Urol Nephrol. 1997;31(1):19-25.

37. Robey EL, Schellhammer PF. The adrenal gland and renal cell carcinoma: Is ipsilateral adrenalectomy a necessary component of radical nephrectomy?. J Urol. 1986;135(3):453-455.

38. Sagalowsky AI, Kadesky KT, Ewalt DM, Kennedy TJ. Factors influencing adrenal metastasis in renal cell carcinoma. J Urol. 1994;151(5):1181-1184.

39. Blake MA, Kalra MK. Current status of imaging for adrenal malignant involvement. Cancer Treat Res. 2008;143:319-329.

40. Lee MJ, Hahn PF, Papanicolaou N, Egglin TK, Saini S, Mueller $\mathrm{PR}$, et al. Benign and malignant adrenal masses: CT distinction with attenuation coefficients, size and observer analisis. Radiology. 1991;179(2):415-418.

41. Gill IS, McClennan BL, Kerbl K, Carbone JM, Wick M, Clayman R. Adrenal involvement from renal cell carcinoma: predictive value of computed tomography. J Urol. 1994;152(4):10821085.

42. Kozak W, Holtl W, Pummer K, Maier U, Jeschke K, Bucher A. Adrenalectomy: Still a must in radical renal surgery?. $\mathrm{Br} \mathrm{J}$ Urol. 1996;77(1):27-31.

Correspondencia autor: Dr. Emilio Ríos González

Servicio de Urología. Hospital Infanta Sofía

Paseo de Europa, 34 - 28702 San Sebastián de los Reyes, Madrid Tel.: 911914138

E-mail autor: erios00@hotmail.com

Información artículo: Original

Trabajo recibido: abril 2008

Trabajo aceptado: mayo 2008 